\title{
A UTILIZAÇÃO DE EQUIPAMENTOS DE PROTEÇÃO INDIVIDUAL ENTRE TRABALHADORES DE ENFERMAGEM DE UM HOSPITAL PÚBLICO*
}

Nathanye Crystal Stanganelli', Renata Perfeito Ribeiro², Caroline Vieira Claudio ${ }^{3}$, Júlia Trevisan Martins², Patricia Helena Vivan Ribeiro ${ }^{4}$, Benedita Gonçalves de Assis Ribeiro ${ }^{5}$

${ }^{1}$ Enfermeira. Residente em Enfermagem Perioperatória. Universidade Estadual de Londrina. Londrina-PR-Brasil. ${ }^{2}$ Enfermeira. Doutora em Enfermagem. Professora da Universidade Estadual de Londrina. Londrina-PR-Brasil. ${ }^{3}$ Enfermeira. Mestranda em Enfermagem. Universidade Estadual de Londrina. Londrina-PR-Brasil.

${ }^{4}$ Enfermeira. Doutora em Enfermagem. Universidade Estadual de Londrina. Londrina-PR-Brasil.

${ }^{5}$ Enfermeira. Mestre em Enfermagem. Professora da Universidade Estadual de Londrina. Londrina-PR-Brasil.

RESUMO: O objetivo foi identificar a utilização dos equipamentos de proteção individual pelos trabalhadores de enfermagem, durante procedimentos que os exponham aos fluidos biológicos. Pesquisa observacional, descritiva, transversal com abordagem quantitativa, desenvolvida em um hospital público do estado do Paraná, no período de janeiro a maio de 2014, utilizando-se da observação não-participante e intencional de 201 procedimentos por meio de um checklist. Verificou-se no Centro Cirúrgico que as luvas de procedimentos (97\%) obtiveram maior adesão, entretanto sapatos fechados e óculos não foram utilizados nenhuma vez. Na Central de Material eEsterilização nenhum trabalhador utilizou as luvas de procedimentos, borracha e térmicas, entretanto a máscara comum $(44,4 \%)$ teve maior aderência na sala de limpeza. Nas Unidades de Terapia Intensiva e Pronto Socorro as luvas de procedimento foram utilizadas (100\%), porém a adesão aos óculos foi baixa $(0,86 \%)$. Os trabalhadores de enfermagem não utilizaram todos os equipamentos preconizados pela legislação durante suas atividades. DESCRITORES: Saúde do trabalhador; Riscos ocupacionais; Prevenção de acidentes; Equipamentos de proteção; Equipe de enfermagem.

\section{THE USE OF INDIVIDUAL PROTECTION EQUIPMENT AMONG THE NURSING STAFF OF A PUBLIC HOSPITAL}

ABSTRACT: The objective was to identify the use of
personal protection equipment by nursing workers
during procedures which expose them to biological
fluids. It was observational, descriptive and transversal
research, with a quantitative approach, undertaken in a
public hospital in the state of Paraná, in January - May
2014 , using nonparticipant and intentional observation
of 201 procedures through the use of a checklist. It was
ascertained that, in the Surgical Center, procedure gloves
(97\%) obtained the greatest adherence, but that closed
shoes and eye protection were not used on any occasion.
In the Central Sterile Services Department, no worker
used the procedure gloves, either rubber or heat-resistant,
although the surgical mask ( $44.4 \%)$ had the greatest
adherence in the cleaning room. In the Intensive Care Units
and Emergency Room, the procedure gloves were used
(100\%), although adherence to the eye protection was low
(0.86\%). The nursing staff did not use all the equipment
stipulated by legislation during their activities.

DESCRIPTORS: Occupational Health; Occupational Risks; Accident Prevention; Protective Devices; Nursing, team.

\section{LA UTILIZACIÓN DE EQUIPOS DE PROTECCIÓN INDIVIDUAL ENTRE TRABAJADORES DE ENFERMERÍA DE UN HOSPITAL PÚBLICO}

RESUMEN: El objetivo de la investigación fue identificar la utilización de los equipos de protección individual por los trabajadores de enfermería, durante procedimientos que los expongan a los fluidos biológicos. Investigación observacional, descriptiva, transversal con abordaje cuantitativo, desarrollada en un hospital público del estado de Paraná, en el periodo de enero a mayo de 2014, utilizándose observación no participante e intencional de 201 procedimientos por medio de un checklist. Se verificó en el Centro Quirúgico que los guantes de procedimientos $(97 \%)$ tuvieron mayor adhesión, sin embargo zapatos cerrados y gafas no fueron utilizados ninguna vez. En la Central de Material y Esterilización, ningún trabajador utilizó guantes de procedimientos, pero la máscara común $(44,4 \%)$ tuvo mayor adherencia en el salón de limpieza. En las Unidades de Terapia Intensiva y Emergencia, los guantes de procedimiento fueron utilizados $(100 \%)$, pero la adhesión a las gafas fue baja $(0,86 \%)$. Los trabajadores de enfermería no utilizaron todos los equipos preconizados por la legislación durante sus actividades. DESCRIPTORES: Salud del trabajador; Riesgos ocupacionales; Prevención de accidentes; Equipos de protección; Equipo de enfermería.

*Artigo extraído do Trabalho de Conclusão de Curso do de Enfermagem intitulado "Utilização de Equipamentos de Proteção Individual em Trabalhadores da Enfermagem: uma avaliação". Universidade Estadual de Londrina, 2014.

Autor Correspondente:

Caroline Vieira Claudio

Universidade Estadual de Londrina

Rua Raposo Tavares, 442 - 86010-580 - Londrina-PR-Brasil

E-mail: caroline.vieirac@gmail.com
Recebido: 04/03/2015

Finalizado: 20/05/2015 


\section{INTRODUÇÃO}

O trabalhador da área da saúde está diariamente exposto aos fatores de riscos ocupacionais, incluindo os químicos, físicos, biológicos, ergonômicos e psicossociais ${ }^{(1)}$. Estes riscos poderão levar o trabalhador ao afastamento precoce das suas atividades e a perda parcial ou total da sua capacidade de exercer a profissão(2).

Uma das maneiras eficazes de minimizar os riscos aos quais os trabalhadores estão expostos, ao exercerem as suas atividades laborais, consiste na utilização correta dos Equipamentos de Proteção Individual (EPIs).

Os EPIs estão descritos na Norma Regulamentadora - 6 (NR-6) como sendo todo dispositivo ou produto de uso individual, utilizado pelo trabalhador e destinado à proteção de riscos que podem ameaçar a sua segurança e saúde. O empregador é obrigado a fornecer aos trabalhadores os equipamentos adequados ao tipo de risco a que estão expostos, em perfeito estado de conservação e funcionamento e de forma gratuita. $\mathrm{O}$ trabalhador deve usar os EPIs de acordo com o risco e a finalidade, adequadamente e responsabilizar-se pela guarda e conservação, bem como comunicar ao empregador qualquer alteração que o torne impróprio para o uso $^{(3)}$.

Com a finalidade de estabelecer as diretrizes básicas para a implementação de ações de proteção à segurança e à saúde dos trabalhadores das instituições de saúde, foi criada a Norma Regulamentadora - 32 (NR-32). Esta norma visa determinar critérios que devem ser seguidos para diminuir os potenciais riscos, melhorando, assim, a qualidade do serviço e da assistência prestada com maior segurança para o trabalhador da saúde ${ }^{(4)}$.

Nas instituições de saúde são desenvolvidas atividades que expõem os trabalhadores aos fluidos biológicos, como o possível contato com secreções corpóreas e até mesmo a ocorrência de acidentes com materiais perfurocortantes. Esta exposição é considerada a mais comum entre os trabalhadores da enfermagem e o tipo mais grave devido ao risco para o desenvolvimento de doenças letais em que mais de 20 tipos de patógenos podem ser transmitidos ${ }^{(5)}$, incluindo o vírus da Imunodeficiência Humana (HIV), o da Hepatite B (HBV) e o da Hepatite C (HCV) ${ }^{(2)}$.
Estudos $^{(6-8)}$ mostram a baixa adesão ao uso de EPIs durante as realizações de procedimentos que envolvam contato com fluidos corporais, incluindo as luvas de procedimentos, luvas estéreis, máscara comum, óculos, dentre outros, ignorando assim, o risco eminente da exposição biológica.

Frente a essas considerações faz-se a seguinte questão de pesquisa: Os trabalhadores de enfermagem de um hospital público utilizam os EPIs necessários durante a assistência de enfermagem? Para responder a esta indagação, tem-se como objetivo identificar a utilização dos EPIs pelos trabalhadores de enfermagem durante a realização de procedimentos que os exponham aos fluidos biológicos.

Tal estudo é de suma importância, visto que identificar a utilização dos EPIs pelos trabalhadores de enfermagem colabora para que reflexões possam ser realizadas com a finalidade de prevenir riscos e agravos, melhorando a qualidade de vida destes trabalhadores.

\section{MÉTODO}

Trata-se de um estudo observacional, descritivo, transversal com abordagem quantitativa, desenvolvido em um hospital público com atendimento de alta complexidade em uma cidade do norte do Paraná, que conta com aproximadamente 903 trabalhadores da saúde.

A coleta dos dados foi realizada no período de janeiro a maio de 2014, por meio da técnica de observação não-participante dos trabalhadores de enfermagem durante a assistência de enfermagem. Foi utilizado um checklist pré-elaborado com os EPIs necessários para cada procedimento realizado nos setores previamente estabelecidos, sendo eles o Centro Cirúrgico (CC), Central de Material e Esterilização (CME), Unidade de Terapia Intensiva I e II (UTI I e UTI II) e Pronto Socorro (PS). Estes cinco setores foram escolhidos por intencionalidade, pois os procedimentos a serem observados ocorrem em frequência e quantidade significativas.

Foram observados por meio do checklist os seguintes procedimentos: no CC, a retirada de materiais das salas cirúrgicas, desprezo dos fluidos corporais dos frascos de aspiração e a utilização do Raio-X em cirurgias ortopédicas; na $\mathrm{CME}$, lavagem de materiais na sala de limpeza, 
acondicionamento e esterilização; no PS, UTI I e II, os curativos com cicatrização por segunda intenção e as aspirações endotraqueais por sistema aberto.

Os setores nos quais foram feitas as observações contam com 248 trabalhadores de enfermagem, sendo observados 201 procedimentos realizados por técnicos e auxiliares de enfermagem nos períodos matutino e vespertino.

Os critérios de inclusão foram os procedimentos realizados pelos enfermeiros, auxiliares e técnicos de enfermagem e como critérios de exclusão os procedimentos realizados por alunos e docentes de enfermagem, outras categorias (fisioterapeutas e médicos) e aqueles realizados com pacientes em isolamentos. No período da coleta nenhum enfermeiro realizou os procedimentos observados, por isso não foram incluídos no estudo.

Os dados coletados foram transcritos por digitação para o programa Microsoft Office Excel $2010 \circledR$ e analisados por meio de estatística descritiva (frequência e percentual) e sob a forma de tabelas. O projeto foi aprovado pelo Comitê de Ética em Pesquisa envolvendo Seres Humanos do local do estudo, sob CAAE $n^{\circ}$ 19911813.3.0000.5231. Todos os preceitos éticos de pesquisa em seres humanos foram atendidos.

\section{RESULTADOS}

A partir dos 201 procedimentos observados nos setores selecionados, na Tabela 1 consta o número de procedimentos e a frequência relativa simples para cada setor, sendo que a maioria dos procedimentos foi observada na UTI I, II e no PS.

No CC, dentre os $51(25,4 \%)$ procedimentos que foram observados, $34(66,7 \%)$ referem-se à

Tabela 1 - Número de procedimentos observados. Londrina-PR-Brasil, 2014

\begin{tabular}{lcc}
\hline Setor & Procedimentos & $\%$ \\
\hline Centro Cirúrgico & 51 & 25,4 \\
\hline $\begin{array}{l}\text { Central de } \\
\text { Material e } \\
\text { Esterilização }\end{array}$ & 29 & 14,4 \\
\hline $\begin{array}{l}\text { Unidade de } \\
\text { Terapia Intensiva } \\
\text { I e II }\end{array}$ & 61 & 30,3 \\
\hline Pronto Socorro & 60 & 29,9 \\
\hline Total & 201 & 100 \\
\hline
\end{tabular}

retirada do material das salas cirúrgicas, seguido pelo desprezo dos fluidos corporais dos frascos de aspiração que consistiram em 10 (19,6\%) e pela utilização do raio-X em cirurgias ortopédicas contando com sete $(13,7 \%)$.

$\mathrm{Na} C \mathrm{ME}$, o total de procedimentos observados foi de $29(14,4 \%)$. Destes, nove (31\%) foram realizados na sala de limpeza (lavagem de materiais), 14 (48,3\%) no acondicionamento e seis $(20,7 \%)$ na esterilização.

$\mathrm{Na}$ UTI I ell, dentre os 61 (30,3\%) procedimentos observados, 59 (96,7\%) correspondem aos curativos por segunda intenção e dois (3,3\%) à aspiração endotraqueal. E no PS, dos 60 (29,9\%) procedimentos observados, 56 (93,3\%) foram os curativos por segunda intenção e quatro $(6,7 \%)$ as aspirações endotraqueais.

Na Tabela 2, pode-se verificar a utilização dos EPIs pelos trabalhadores de enfermagem, bem como a frequência relativa, durante os procedimentos observados no CC, CME, UTI I, UTI II e PS.

Como podemos constatar por meio da Tabela 2, dos 34 procedimentos observados no CC, durante a retirada do material das salas operatórias, as luvas de procedimento foram utilizadas pela maioria dos trabalhadores de enfermagem (97\%) e os sapatos fechados apenas em cinco (14,7\%). Em relação ao desprezo dos fluidos corporais, quatro $(40 \%)$ dos 10 procedimentos observados foram utilizadas as máscaras comuns. Entretanto, as luvas de borracha, os óculos e os sapatos fechados não foram utilizados nenhuma vez. Os aventais de chumbo, no CC, foram utilizados em três (42,9\%) dos sete procedimentos realizados com a utilização do Raio-X.

$\mathrm{Na} C M E$, especificamente na sala de limpeza, na qual se realiza a lavagem dos materiais, foi observada a utilização da máscara comum em quatro $(44,4 \%)$ dos nove procedimentos. Porém, as luvas de borracha, sapatos fechados e os óculos não foram utilizados nenhuma vez. Na área de acondicionamento dos materiais foi utilizada a máscara comum apenas uma vez $(7,1 \%)$ dos 14 procedimentos observados e nenhuma vez as luvas de procedimento. E na área de esterilização as luvas térmicas e o protetor auricular não foram utilizados nenhuma vez.

Em relação aos 115 procedimentos de curativos observados, foi constatada a utilização das luvas de procedimento em todos os trabalhadores, entretanto os óculos foram utilizados apenas uma vez $(0,86 \%)$. Durante as aspirações endotraqueais, os óculos foram utilizados apenas duas vezes (33,3\%). 
Tabela 2 - Utilização dos Equipamentos de Proteção Individual pelos trabalhadores de enfermagem do CC, CME, UTI I, UTI II e PS. Londrina-PR-Brasil, 2014

\begin{tabular}{|c|c|c|c|c|}
\hline Procedimento & Frequência absoluta & EPI & Utilização dos EPI & Frequência relativa \\
\hline \multirow{2}{*}{$\begin{array}{l}\text { Retirada dos } \\
\text { materiais }\end{array}$} & \multirow[t]{2}{*}{34} & $\begin{array}{l}\text { Luvas de } \\
\text { procedimento }\end{array}$ & 33 & $97 \%$ \\
\hline & & Sapatos fechados & 5 & $14,7 \%$ \\
\hline \multirow{4}{*}{$\begin{array}{l}\text { Desprezo dos fluidos } \\
\text { corporais }\end{array}$} & \multirow{4}{*}{10} & Máscaras comuns & 4 & $40 \%$ \\
\hline & & Luvas de borracha & 0 & - \\
\hline & & Óculos & 0 & - \\
\hline & & Sapatos fechados & 0 & - \\
\hline Utilização do Raio-X & 7 & Avental de chumbo & 3 & $42,9 \%$ \\
\hline \multirow{4}{*}{ Sala de limpeza } & \multirow{4}{*}{9} & Luvas de borracha & 0 & - \\
\hline & & Sapatos fechados & 0 & - \\
\hline & & Óculos & 0 & - \\
\hline & & Máscaras comuns & 4 & $44,40 \%$ \\
\hline \multirow[t]{2}{*}{ Acondicionamento } & \multirow[t]{2}{*}{14} & $\begin{array}{l}\text { Luvas de } \\
\text { procedimento }\end{array}$ & 0 & - \\
\hline & & Máscaras comuns & 1 & $7,10 \%$ \\
\hline \multirow[t]{2}{*}{ Esterilização } & \multirow[t]{2}{*}{6} & $\begin{array}{l}\text { Luvas de } \\
\text { procedimento } \\
\end{array}$ & 0 & - \\
\hline & & Máscaras comuns & 1 & $7,10 \%$ \\
\hline \multirow[t]{2}{*}{ Curativo } & \multirow[t]{2}{*}{115} & $\begin{array}{l}\text { Luvas de } \\
\text { procedimento }\end{array}$ & 115 & $100 \%$ \\
\hline & & Óculos & 1 & $0,86 \%$ \\
\hline $\begin{array}{l}\text { Aspiração } \\
\text { endotraqueal }\end{array}$ & 6 & Óculos & 2 & $33,3 \%$ \\
\hline
\end{tabular}

Legenda: EPI: Equipamentos de Proteção Individual

\section{DISCUSSÃO}

Neste estudo foi possível observar que durante os 34 procedimentos de retirada do material das salas cirúrgicas, apenas cinco $(14,7 \%)$ trabalhadores de enfermagem utilizaram os sapatos fechados. Entretanto, a maioria (97\%) utilizou as luvas de procedimentos na execução dessa tarefa, diferenciando-se de outro estudo realizado com circulantes da sala cirúrgica, no qual se verificou que os trabalhadores não utilizavam os EPIs preconizados, como as luvas de procedimentos durante a retirada de compressas sujas de sangue ${ }^{(9)}$.

Salienta-se que o preconizado durante a retirada dos materiais das salas cirúrgicas é o uso dos seguintes EPIs: luvas de procedimento devido ao contato com fluidos biológicos e umidade proveniente de operações com uso de água, além da máscara comum e sapatos fechados com a utilização dos propés ${ }^{(10)}$.

Em relação aos 10 procedimentos observados durante o desprezo dos fluidos corporais dos frascos de aspiração utilizados durante os procedimentos cirúrgicos, o uso da máscara comum teve baixa aderência entre os trabalhadores $(40 \%)$ e as luvas de procedimentos, óculos e sapatos fechados não foram utilizadas nenhuma vez neste estudo. A baixa adesão ao uso dos EPIs pode estar relacionada a fatores como desconforto, incômodo e inadequação ${ }^{(11)}$.

A preocupação é grande em relação à baixa adesão do uso dos EPIs durante o desprezo dos fluidos corporais, pois na maioria das vezes, esses frascos contém quantidade significativa de fluidos que podem provocar respingos durante o seu desprezo e se configurar potencial contaminante.

Tratando-se do uso do Raio-X nas salas cirúrgicas, todos os trabalhadores expostos a radiação ionizante devem proteger-se dessa radiação através do uso de vestimenta ou barreiras protetoras com atenuação não inferior a $0,25 \mathrm{~mm}$ equivalentes de chumbo ${ }^{(12)}$. De acordo com os resultados do presente estudo, não houve 
aderência significativa ao uso deste EPI, pois apenas três $(42,9 \%)$ trabalhadores utilizaram. Em contrapartida, outro estudo constatou que $100 \%$ dos trabalhadores utilizaram este EPI devido à utilização de Raio-X em salas de hemodinâmica ${ }^{(13)}$.

A baixa adesão ao uso de vestimentas com chumbo, no presente estudo, pode estar relacionada com as características deste $\mathrm{EPI}^{(14)}$, ou seja, a presença do chumbo faz com que este EPI seja pesado, gerando desconforto e até dores lombares após a sua utilização ${ }^{(13)}$.

$\mathrm{Na} C M E$, especificamente na sala de limpeza, os materiais necessitam ser limpos, sendo necessária a utilização de máscara comum, luvas longas de borracha, avental impermeável de manga longa, protetor auricular (se necessário), óculos e sapato fechado impermeável e antiderrapante. Os óculos e a máscara comum podem ser substituídos pelo protetor facial. Esses EPIs protegem os trabalhadores frente à exposição aos fluidos biológicos e à umidade ${ }^{(15)}$. Entretanto, neste estudo nenhum trabalhador utilizou os sapatos fechados, óculos e avental descartável na sala de limpeza, revelando a baixa aderência.

Verificou-se também que os trabalhadores de enfermagem não utilizam as luvas de borracha para a lavagem dos materiais na sala de limpeza, preferindo as luvas de procedimento que propiciam menor proteção em relação a riscos de acidentes com materiais perfurocortantes e à umidade. Em um estudo que avaliou os riscos e os mecanismos de auto cuidado dos trabalhadores da $\mathrm{CME}$, os mesmos referiram que as luvas de borracha retiram a sensibilidade e dificultam o manuseio dos materiais ${ }^{(16)}$.

$\mathrm{Na}$ década de 90, com a introdução dos limpadores enzimáticos na limpeza eficiente dos materiais contaminados na sala de limpeza, houve a discussão da necessidade ou não do uso de luvas de procedimentos durante o acondicionamento dos artigos, já que a limpeza de forma eficiente dos materiais contaminados pode reduzir a carga microbiana de maneira significativa ${ }^{(17)}$, deixando o material seguro para o manuseio. Porém, resolução brasileira institui como obrigatório o uso das luvas de procedimentos, máscara e protetor auricular ${ }^{(15)}$ para tal atividade. No presente estudo, nenhum trabalhador de enfermagem utilizou as luvas de procedimento e apenas um (7,1\%) utilizou a máscara comum.
Durante a esterilização, embora a resolução brasileira não aborde sobre a necessidade do uso de EPI nesta área ${ }^{(15)}$, devem-se utilizar as luvas térmicas para a retirada dos materiais da autoclave, as quais evitam queimaduras no trabalhador, bem como óculos de proteção, máscara comum, sapatos fechados e avental impermeável ${ }^{(10)}$. Entretanto, no presente estudo verificou-se que os trabalhadores de enfermagem não fizeram uso das luvas térmicas, utilizando apenas para se proteger retalhos de tecidos que estavam próximos ao equipamento, expondo-os ao risco de queimaduras devido à alta temperatura dos materiais e das autoclaves.

O protetor auricular também deve ser utilizado na esterilização, pois os trabalhadores de enfermagem estão expostos aos ruídos dos equipamentos de esterilização que, sem a devida utilização de protetores auriculares, as altas frequências sonoras podem prejudicar a saúde destes trabalhadores.

Em estudo realizado para verificar os níveis de ruído em um setor de esterilização, constatou que estes níveis estavam elevados, pois a média foi de 66 decibéis ${ }^{(18)}$, sendo que os níveis aceitáveis de ruídos são de 34 a 45 decibéis ${ }^{(19)}$. Embora não tenham sido verificados os níveis de decibéis a que os trabalhadores do presente estudo estiveram expostos, infere-se que seja necessário o uso destes EPIs, entretanto, no presente estudo nenhum trabalhador os utilizou.

Em relação ao uso dos EPIs para a realização de curativos com cicatrização por segunda intenção, nos quais a limpeza da ferida é realizada pelo método de irrigação, faz-se necessário o uso dos seguintes EPIs: máscara comum, avental, óculos e luvas de procedimentos ${ }^{(20)}$. Ressalta-se que os curativos foram realizados por meio de pinças estéreis, dispensando o uso de luvas estéreis.

Neste estudo, a máscara comum (70,4\%) e as luvas de procedimentos (100\%) tiveram adesão significativa durante a realização de curativos com cicatrização por segunda intenção. Entretanto, os óculos foram utilizados apenas uma vez $(0,86 \%)$ pelos trabalhadores durante a realização dos 115 curativos observados. Este dado se assemelha ao resultado de outro estudo, o qual evidenciou que menos da metade dos trabalhadores utilizavam óculos para a realização do curativo ${ }^{(21)}$. 
Com relação às aspirações endotraqueais pelo sistema aberto, faz-se necessário o uso de máscara comum, óculos para proteção contra possíveis partículas voláteis e luvas estéreis para proteção do paciente e do trabalhador, além de avental descartável ${ }^{(22)}$.

Este estudo constatou que a máscara comum, luvas estéreis e os aventais descartáveis foram utilizados por todos os trabalhadores de enfermagem observados durante as aspirações endotraqueais. Entretanto, apenas dois (33,3\%) trabalhadores utilizaram os óculos de proteção. Complementando, outro estudo verificou que dos 334 procedimentos de aspiração das vias aéreas superiores observados, apenas em 25 (7,8\%) foram utilizados todos os EPls necessários ${ }^{(7)}$.

Um estudo constatou os motivos pelos quais os trabalhadores não utilizam os EPIs durante os procedimentos: sensação de sufoco, incômodo, desconforto, dificuldade na utilização, calor e obrigatoriedade da utilização(11). Esse comportamento de não utilizar os EPIs de maneira correta influencia direta e indiretamente na segurança dos trabalhadores, colocando-os em situação de risco ${ }^{(23)}$.

O trabalhador da área da saúde, incluindo a enfermagem, necessita conhecer os riscos aos quais está exposto. Portanto, faz-se necessário realizar educação permanente para que medidas sejam adotadas, prevenindo, assim, os riscos e melhorando a qualidade de vida dos trabalhadores.

Autores ${ }^{(13,24-25)}$ afirmam que é preciso aprendizagem significativa para que as práticas de precauções padrão sejam adotadas de maneira eficaz, promovendo assim, prevenção de acidentes e doenças ocupacionais, sendo essencial a adoção de medidas preventivas para a melhoria da saúde do trabalhador.

Complementarmente, é de fundamental importância que os trabalhadores de enfermagem compreendam primeiramente quais são os riscos ocupacionais a que estão expostos e, assim, possam ficar atentos a estes riscos a fim de evitálos e proteger a saúde dos mesmos ${ }^{(26)}$.

\section{CONCLUSÃO}

Pode-se afirmar que os trabalhadores de enfermagem investigados não utilizam corretamente todos os EPIs necessários para os procedimentos realizados, os quais são preconizados pela legislação brasileira.

Constatou-se no CC que as luvas de borracha, os óculos de proteção e os sapatos fechados não foram utilizados por nenhum trabalhador. Entretanto, as luvas de procedimentos obtiveram significativa adesão. Na CME nenhum trabalhador utilizou as luvas de procedimento, borracha e térmicas necessárias, além dos sapatos fechados, óculos e aventais impermeáveis, sendo a máscara comum o equipamento que obteve maior adesão. Nas UTI e no PS a adesão aos óculos foi muito baixa, ao contrário das luvas de procedimento que foram utilizadas por todos os trabalhadores.

Embora o objetivo do estudo tenha sido alcançado, o estudo apresentou limitações por se tratar de um estudo transversal o qual não permite a generalização dos achados pesquisados. Assim, sugere-se que novas pesquisas sejam desenvolvidas, incluindo a utilização dos EPIs em outros procedimentos e setores que não foram abordados neste estudo, bem como o efeito dos treinamentos nos serviços de saúde.

\section{REFERÊNCIAS}

1. Marziale MHP. Ocurrencia de accidentes de trabajo causados por material corto-punzante entre trabajadores de enfermería em hospitales de la región nordeste de São Paulo, Brasil. Cienc. enferm. [Internet] 2003;9(1) [acesso em 01 set. 2014]. Disponível: http:// dx.doi.org/10.4067/S0717-95532003000100004

2. Ministério da Saúde (BR). Exposição a materiais Biológicos. Brasília: Editora Ministério da Saúde; 2006.

3. Ministério do Trabalho e Emprego (BR). Portaria n. 25, de 15 de outubro de 2001. Altera a Norma Regulamentadora que trata de Equipamento de Proteção Individual - NR6 e dá outras providências. Diário Oficial da República Federativa da União, Brasília, 17 out. 2001. Seção 1:1.

4. Ministério do Trabalho e Emprego (BR). Portaria n. 485, de 11 de novembro de 2005. Aprova a Norma

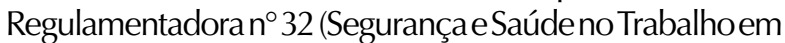
Estabelecimentos de Saúde). Diário Oficial da República Federativa da União, Brasília, 16 nov. 2005. Seção 1:1.

5. Sarquis LMM, Felli VEA. Orientações aos trabalhadores de enfermagem diante da exposição aos fluidos biológicos. In: Martini JG, Felli VEA, organizadores. Porto Alegre: Artmed/Panamericana; 2009. p.9-38.

6. Freiberger MF, Correia MBR, Pinto EAM, Ferreira EJ. 
Adesão ao uso de óculos de proteção individual pelos profissionais de saúde em unidade de Centro cirúrgico. Rev Cie Fac Edu Mei Amb. 2011;2(2):70-9.

7. Farias GM, Freire ILS, Ramos CS. Aspiração endotraqueal: estudo em pacientes de uma unidade de urgência e terapia intensiva de um hospital da região metropolitana de Natal - RN. Rev. Eletr. Enf. [Internet] 2006;8(1) [acesso em 25 nov. 2014]. Disponível: http:// www.fen.ufg.br/revista/revista8_1/original_08.htm

8. Oliveira AC, Machado BCA, Gama CS. Conhecimento e adesão às recomendações de biossegurança no Corpo de Bombeiros Militar de Minas Gerais. Rev. esc. enferm. USP [Internet] 2013;47(1) [acesso em: 10 jan 2015]. Disponível: http://dx.doi.org/10.1590/ S0080-62342013000100015

9. Almeida ANG. Risco biológico para circulante de sala operatória [dissertação]. Goiânia (GO): Universidade Federal de Goiás; 2009.

10. Ministério da Saúde (BR). Doenças relacionadas ao trabalho: manual de procedimentos para o serviço de saúde. Brasília: Editora Ministério da Saúde; 2001.

11. Ribeiro RP, Vianna LAC. Uso de equipamentos de proteção individual entre trabalhadores das centrais de material e esterilização. Ciênc. cuid. saúde [Internet] 2012;11 Suppl [acesso em: 01 set 2014]. Disponível: http://dx.doi.org/10.4025/cienccuidsaude.v11i5.17076

12. Ministério da Saúde (BR). Portaria n. 453 , de $1^{\circ}$ de junho de 1998. Aprova as diretrizes de proteção radiológica em radiodiagnóstico médico e odontológico. Diário Oficial da República Federativa da União, Brasília, 2 jun. 1998.

13. Gallo AM, Lima FAC, Reis LM, Cremer E. Exposição ocupacional à radiações ionizantes sob a ótica de profissionais de enfermagem em hemodinâmica. Rev Rene [Internet]. 2013;14(1) [acesso em 01 nov. 2014]. Disponível: http://www.revistarene.ufc.br/revista/ index.php/revista/article/view/285

14. Soares FAP, Pereira AG, Flôr RC. Utilização de vestimentas de proteção radiológica para redução de dose absorvida: uma revisão integrativa de literatura. Radiol Bras [Internet] 2011;44(2) [acesso em 01 nov. 2014]. Disponível: http://dx.doi.org/10.1590/S010039842011000200009

15. Ministério da Saúde (BR). Agência Nacional de Vigilância Sanitária. Resolução da Diretoria Colegiada n. 15, de 15 de março de 2012. Dispõe sobre requisitos de boas praticas para processamento de produtos para a saúde e da outras providências. Diário Oficial da República Federativa da União, Brasília, 19 mar. 2012.

16. Espindola MCG, Fontana RT. Riscos ocupacionais e mecanismos de autocuidado do trabalhador de um centro de material e esterilização. Rev. Gaúcha Enferm. [Internet] 2012;33(1) [acesso em 20 set.
2014]. Disponível: http://dx.doi.org/10.1590/S198314472012000100016

17. Sociedade Brasileira de Enfermeiros de Centro Cirúrgico, Recuperação Anestésica e Centro de Material e Esterilização (SOBECC). Práticas recomendadas da SOBECC. 6 $6^{a}$ ed. São Paulo: SOBECC; 2013.

18. Otenio MH, Cremer E, Claro ET. Intensidade de ruído em hospital de 222 leitos na $18^{\text {a }}$ Regional de Saúde PR. Rev. Bras. Otorrinolaringol. [Internet] 2007;73(2) [acesso em 07 nov. 2014]. Disponível: http://dx.doi. org/10.1590/S0034-72992007000200016

19. Associação Brasileira de Normas Técnicas (ABNT). Norma brasileira 10152: Avaliação do ruído para o conforto acústico. Rio de Janeiro: ABNT; 1987.

20. Guariente MHDM. Técnicas de Enfermagem. Pontos RelevantesnoEnsinarenoCuidar.Londrina:EditoraEduel;2011.

21. Bezerra VNP, Pereira MLD. Utilização de equipamento de proteção individual pela equipe de enfermagem da unidade clinico-cirúrgica. Rev Rene [Internet] 2004;5(1) [acesso em 05 nov. 2014]. Disponível: http://www.revistarene. ufc.br/revista/index.php/revista/article/view/847

22. Hinrichsen SL. Aspectos éticos e jurídicos dos processos infecciosos hospitalares e suas relações com a vigilância e a qualidade assistencial. Rio de Janeiro: Medsi; 2004. Biossegurança e controle de infecções: risco sanitário hospitalar. p. 273-81.

23. Mendonça KM, Tipple AFV, Rezende FR, Souza ACS, Pereira MS. Risco biológico nas etapas finais do sistema de medicação nos setores de urgência e emergência. Rev. Eletr. Enf. [Internet] 2014;16(3) [acesso em 04 nov 2014]. Disponível: http://dx.doi. org/10.5216/ree.v16i3.27436

24. Alan MM, César-Vaz MR, Almeida T. Educação ambiental e o conhecimento do trabalhador em saúde sobre situações de risco. Ciênc. saúde coletiva [Internet] 2005;10 Suppl [acesso em 02 dez. 2014]. Disponível: http://dx.doi.org/10.1590/S141381232005000500007

25. Ribeiro PHV, Gir E, Santos CB, Ribeiro RP, Hayashida $M$, Malaguti-Toffano SE, et al. Validation of an instrument to assess factors associated with adherence to standard precautions among dentists in primary healthcare. Acta odontol. latinoam. [Internet] 2014;27(1) [acesso em 02 nov. 2014]. Disponível: http://www.scielo.org.ar/scielo.php?pid=S185248342014000100002\&script=sci_arttext

26. Giovelli G, Cardoso SMM, Fontana RT, Rodrigues FCP, Brum ZP. A percepção dos técnicos de enfermagem em relação aos riscos ocupacionais em salas de vacinas. Cogitare enferm. [Internet] 2014;19(2) [acesso em 10 jan. 2015]. Disponível: http://dx.doi. org/10.5380/ce.v19i2.37006 